\title{
Ontology-based Approach for Hepatobiliary System Diseases
}

\author{
Galal AL Marzoqi, Marco Alfonse, Ibrahim F. Moawad, Mohamed Roushdy \\ Faculty of Computer and Information Science, Ain shams University, Abbasia, Egypt
}

\begin{abstract}
Medical Ontologies play a central role in integrating heterogeneous databases of various model organisms. The Hepatobiliary system is very important for human vital processes. It has an ability to regulate the other systems. Furthermore, it plays an important role in many body functions like protein production. Also, it may be affected by many pathologic conditions, which affect other organs negatively.. This paper investigates the current studies on Ontology-based approach and Ontology techniques for Hepatobiliary System Diseases. We present conceptual view for the Hepatobiliary system and its infected diseases. Besides, we propose a new classification schema for the research efforts investigated so far. We classified the research efforts investigated so far based on the Hepatobiliary system organs: Liver, Gallbladder, Bile duct and Pancreas. Besides, we discuss the current research gaps found in this research area.
\end{abstract}

\section{Introduction}

Ontology is a kind of controlled vocabulary of well-defined terms with specified relationships between those terms, capable of interpretation by both humans and computers [1]. Furthermore, it is a specific rich description of Terminology, Rules, Concepts, and Relations among the concepts. There are several Ontology languages such as Extensible Markup Language (XML) [2], Resource Description Framework Schema $(\operatorname{RDF}(\mathrm{S}))$ [3], Darpa Agent Markup Language Ontology Interface Language (DAML+OIL) [4], and Web Ontology Language (OWL) [5]. The Ontologies can be exploited in many applications in fields, where semantics-based communication among people and systems are crucial. [6]. Also, there are different techniques related to the Ontologies: Ontology alignment, Ontology mapping/matching, Ontology translation, Ontology merging/integrating Ontology refinement and Ontology unification [7]. Ontology tools can be applied to all stages of the Ontology lifecycle (creation, population, validation, deployment, maintenance, and evolution), and hence there are many tools for Ontologies management in different formats (Protégé, OilEd, Apollo, RDFedt, OntoLingua, OntoEdit, WebODE, KAON, ICOM, DOE, and WebOnto) [8].
Ontology can be used to support various knowledge management issues, including knowledge retrieval, storing, and sharing [9]. Protégé is open source software that provides the user community with a suite of tools to construct domain models and knowledge-based applications with Ontologies Protégé implements a rich set of knowledgemodeling structures and actions that support the creation, visualization, and manipulation of Ontologies in various representation formats. Protégé can be customized to provide domain for creating knowledge models and entering data [10].

Medical Ontologies play a central role in integrating heterogeneous databases of various model organisms and stored in heterogeneous databases. Also, it defines a precise and shared vocabulary for the semantic markup of resources and their description by metadata [11]. Conceptually, it is interested in solving important issues such as the reusing and sharing of medical data. The unambiguous communication of complex and detailed medical concepts is now a crucial feature of medical information systems [12]. There are Medical Ontologies developed to facilitate this purpose, such as (Open Biomedical Ontologies OBO [13], National Center for Biomedical Ontology NCBO's BioPortal [14] and Unified Medical Language System UMLS [15]). Medical Ontology is now widely acknowledged that Ontologies can make a significant contribution to the design and implementation of information systems in the medical field.

On the other hand, human body systems consist of specific cells, tissues, and organs that work together to perform specific functions. Conceptually, these systems are interconnected and dependent, so they can't work separately, such as (Nervous System, Respiratory System, Immune System, Digestive System and Hepatobiliary System) [16,17]. The Hepatobiliary system includes four organs (Liver, Gallbladder, Bile duct and Pancreas). Where, Hepatobiliary is the one of the important systems in the human body. It is responsible for lots of processes inside the body. These processes are important to keep body regulated and healthy. Conceptually, it plays an important role in many body functions like protein production. It is also responsible for detoxification, metabolism, synthesis, and storage of various substances, however, this vital system may be affected by hazardous conditions 
whether they are internal or external [18, 19]. Interestingly, these diseases were classified based on different dimensions such as (Cause, Treatment, Symptoms, etc.). This paper presents a new classification schema for the research efforts investigated so far. We classified the research efforts investigated so far based on the Hepatobiliary system organs: Liver, Gallbladder, Bile duct and Pancreas.

The paper is organized as follows. Section 2 presents the conceptual view of Ontology based Hepatobiliary Systems. Section 3 displays Liver based Systems, section 4 presents Gallbladder based System, section 5 presents Pancreas based Systems, and section 6 presents Comparative among Ontology-based systems for Hepatobiliary System Diseases. Finally, section 7 presents conclusion.

\section{Conceptual View Ontology Based Hepatobiliary Systems}

In the general human body consists of many systems such as (Nervous System, Respiratory System, Immune System, Digestive System and Hepatobiliary System). The Hepatobiliary system includes four organs (Liver, Gallbladder, Bile duct and Pancreas). To understand the research paper so far Hepatobiliary system, we design conceptual view for human body and its related systems.

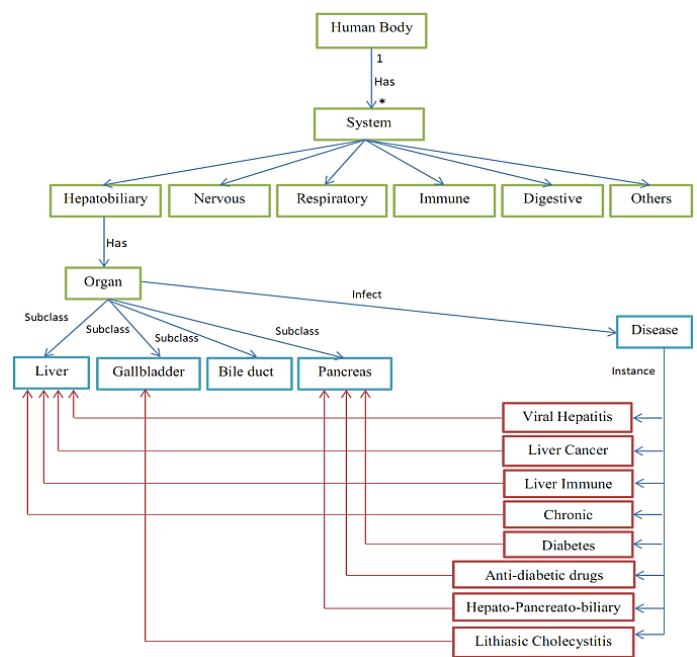

Figure 1. Conceptual view of Hepatobiliary System

Figure 1 shows a new classification schema in the "Human Body" is the main class. Also, we have the "System" class. The "System" class into six subclasses which is "Nervous", "Respiratory", "Immune", "Digestive", "Hepatobiliary" and "Others". The "Hepatobiliary" class has "Organ" class. The "Organ" class includes five subclasses: "Liver", "Gallbladder", "Bile duct", "Pancreas" and "Disease". The "Disease" class includes eight instances: "Viral Hepatitis", "Liver Cancer", "Liver Immune", "Chronic", "Diabetes", "Anti-diabetic drugs", "Hepato-Pancreato-biliary" and "Lithiasic Cholecystitis" which have effect on organs: Liver, Gallbladder, Bile duct and Pancreas in Hepatobiliary system.

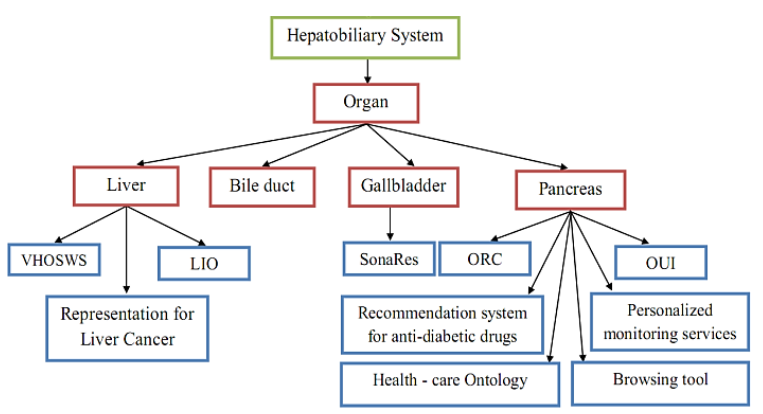

Figure 2. Ontology Based Systems for Hepatobiliary System Diseases

There are many domains specific Ontology-based approach and Ontology techniques have been built on Hepatobiliary System Diseases. Figure 2 shows the research efforts investigated so far based on the Hepatobiliary system organs: Liver, Gallbladder, Bile duct and Pancreas.

\section{Liver Based Systems}

In [20], the authors presented the architecture of the proposed Viral Hepatitis Ontology Sharing Web Service approach. The main module of this approach is the Viral Hepatitis Ontology sharing Web Service that accesses the Viral Hepatitis OWL Ontology to share its primitives (concepts and properties) among physicians, students of medicine, and intelligent systems. The Viral Hepatitis Ontology Sharing Web Service includes the service operations needed for Ontology sharing and Viral Hepatitis diseases diagnosing. The OWL Viral Hepatitis Ontology contains the Viral Hepatitis diseases and their signs, symptoms, and laboratory-findings. To exploit the Viral Hepatitis Ontology Sharing Web Service, physicians, students of medicine, and intelligent systems can invoke the web service operations through the internet in different usage scenarios.

\subsection{The Viral Hepatitis Ontology}

OBR-based OWL Ontology for Viral Hepatitis (A, B, C and D) was developed. The bottom-up approach has been exploited in designing this Ontology. Because the Viral Hepatitis A, B, C and D viruses are the most widely spread among males and females, this Ontology can be shared, and exploited in different ways by both Intelligent Systems and Physicians. The Ontology is represented in Web Ontology Language (OWL) that has become recently the standard language for the semantic web. The protégé-OWL editor was used to implement the 
Ontology.

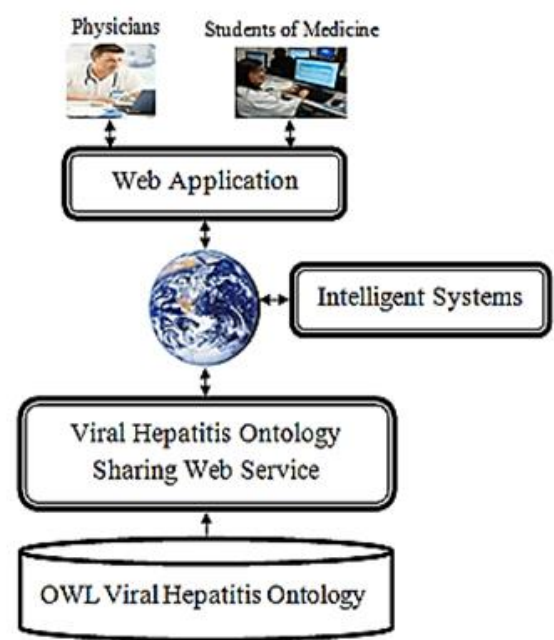

Figure 3. The Approach Architecture [20]

\subsection{Web Service Operations}

To achieve the Viral Hepatitis Ontology sharing and Viral Hepatitis diseases diagnosing objectives of this research, the web service should contain set of operations that can be invoked through the internet in different usage scenarios. By analyzing the approach requirements, they found that twelve web service operations should be provided. Table 1 shows sample of those operations in terms of operation description, inputs and outputs.

Table 1. The sample of Web Service Operations [20]

\begin{tabular}{|c|c|c|c|}
\hline Web Service Operation & Description & Input & Output \\
\hline ViewHierarchicalViral HepatitisOntology & $\begin{array}{l}\text { Returns the whole Viral } \\
\text { Hepatitis Ontology file. }\end{array}$ & & OWL File \\
\hline QueryForCommonDiseases OfSymptomsOrSings & $\begin{array}{l}\text { Returns list of the common } \\
\text { diseases for symptoms or } \\
\text { sings. }\end{array}$ & $\begin{array}{l}\text { List of symp- } \\
\text { toms or sings }\end{array}$ & $\begin{array}{l}\text { List of diseas- } \\
\text { es }\end{array}$ \\
\hline RetrieveCommonDiseasesForSymptoms & $\begin{array}{l}\text { Returns list of diseases caus- } \\
\text { ing the input symptoms. }\end{array}$ & $\begin{array}{l}\text { List of symp- } \\
\text { toms }\end{array}$ & $\begin{array}{l}\text { List of diseas- } \\
\text { es }\end{array}$ \\
\hline RetrieveCommonDiseasesForSigns & $\begin{array}{l}\text { Returns list of diseases caus- } \\
\text { ing the input signs. }\end{array}$ & List of signs & $\begin{array}{l}\text { List of diseas- } \\
\text { es }\end{array}$ \\
\hline QueryFor SymptomsSingsOrLaboratory-findings & $\begin{array}{l}\text { Returns symptoms, signs and } \\
\text { laboratory-findings for a } \\
\text { specific disease. }\end{array}$ & A disease & $\begin{array}{l}\text { List of symp- } \\
\text { toms, sings, } \\
\text { and laborato- } \\
\text { ry-findings }\end{array}$ \\
\hline Retrie veSymptomsForDisease & $\begin{array}{l}\text { Returns a list of symptoms for } \\
\text { a specific disease. }\end{array}$ & A disease & $\begin{array}{l}\text { List of symp- } \\
\text { toms }\end{array}$ \\
\hline
\end{tabular}

\subsection{Prototype Implementation}

As shown in the approach architecture, the prototype consists of two main components: the Viral Hepatitis Ontology sharing web service and a web application that lets physicians and students of medicine and Intelligent Systems benefit this web service. The web service component was developed using the C\# language (one of the Microsoft Visual Studio .NET programming languages), and the web application was developed using the ASP.NET (Active Server Pages Technology).

The home page of the web application contains four menu items: View Hierarchical Ontology, Query for Diseases, Query for Symptoms and Signs, and Diagnose Viral Hepatitis. In the following, sample of different usage case studies for the system prototype are presented. In figure 4 the VHOSWS tool for Viral Hepatitis differential diagnosis is shown.

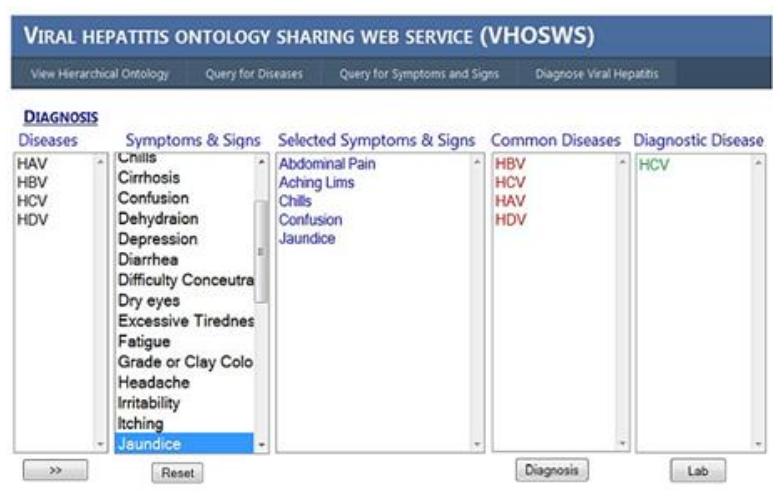

Figure 4. Viral Hepatitis Differential Diagnosis [20]

The goal of this research, a new Web Service based approach to share the Viral Hepatitis Ontology among physicians, students of medicine, and intelligent systems was presented. The approach enables physicians and students of medicine to differentially diagnose the Viral Hepatitis diseases. In addition, a system prototype (VHOSWS) was developed to show how the approach is very beneficial for physicians and students of medicine by presenting different usage case studies.

In [21], the authors developed Liver cancer Ontology was built by Protégé-OWL editor [22, 23]. Primary liver cancer is cancer that begins in the liver. The subtypes of primary liver cancer are named for the type of cell from which they develop. There are three subtypes of primary liver cancer namely, Hepatocellular carcinoma, Cholangiocarcinoma, Angiosarcoma. For more information about the medical description see [24]. The liver cancer is described in terms of its risk factors, symptoms, diagnosis, staging and treatment [25].

\subsection{Liver Cancer Ontology Classes and Instances}

The Class hierarchy of the liver cancer Ontology from this figure, it can be seen that, the liver cancer Ontology has three main classes; Disease, Medical Intervention and References. The Disease class contains the Liver Cancer class with its types. The Medical Intervention class contains the Staging, Diagnosis and Treatment classes. The references class contains the Symptoms, Stage, Risk Factors and TNM_System classes. Each one of these classes may have its own subclasses according to the structure of the liver cancer. Figure 5 shows the class hierarchy of the Liver Cancer Ontology.

The instances of classes as described in the liver cancer Ontology. The classes M, N and T are defined as enumerated classes. Each one of these classes is described in terms of its instances (no more instances 
can be added to any of these classes) as shown in Figure 6.

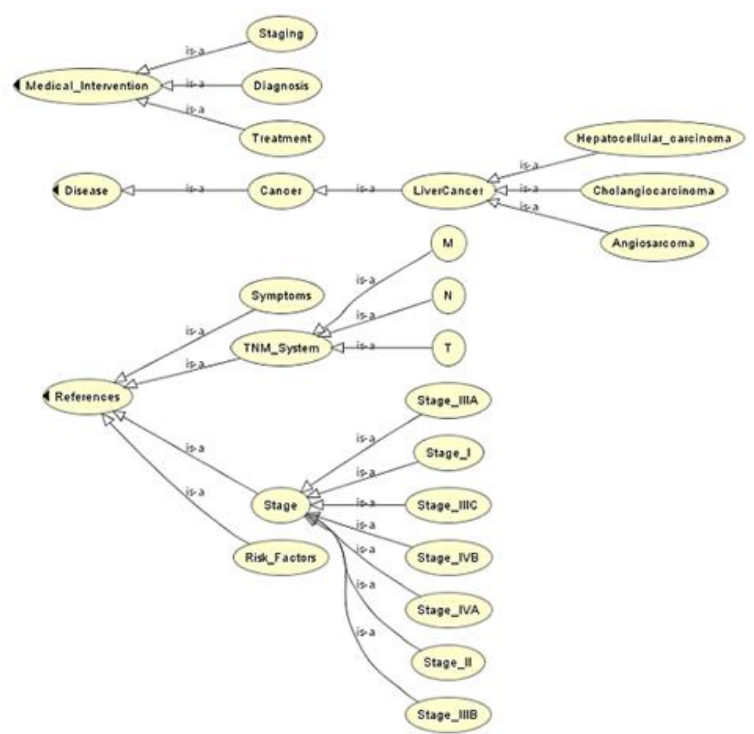

Figure 5. Liver Cancer Class Hierarchy [21]

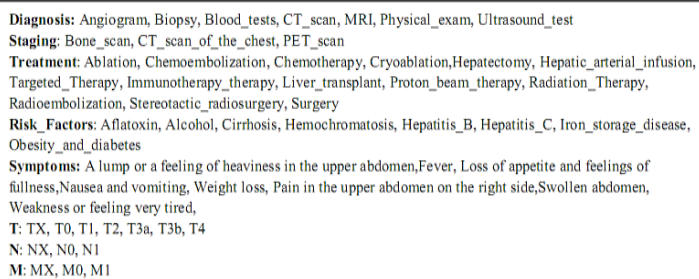

Figure 6. The Instances of the Classes of the Liver Cancer Ontology [21]

The goal of this research is to develop Ontology based knowledge representation for Liver Cancer that was built using the Protégé-OWL editing environment. It has a great user interface that eases the process of building or editing Ontologies. This Ontology is encoded in OWL-DL format which is the most recent development in standard Ontology languages, endorsed by the World Wide Web Consortium (W3C) to promote the Semantic Web vision. This Ontology can be used by experts or medical researchers who want the liver cancer knowledge to be represented in a semantic way that allows reasoning capabilities.

In [26], the authors developed Liver Immunology Ontology (LIO) within the Open Biomedical Ontologies (OBO) Foundry framework, importing and linking relevant portions of orthogonal reference Ontologies. LIO is a novel tool for comprehensive analysis of liver immunology data sets, providing a valuable resource for the liver disease research community.

\section{Gallbladder Based System}

In [27], the authors presented a description of the process of Ontology construction for gallbladder ultrasound images. This Ontology is inspired and based on the knowledge base created and being used for SonaRes- the decision support system for ultrasound diagnostics [28, 29]. This system has accumulated the experience of the skillful expertssonographists in the domain of hepato-pancreatobiliary zone examination.

This experience and knowledge are well structured and formalized in this system for gallbladder and pancreas. On the other hand, there is a powerful and attractive, from the point of view of knowledge portability, tool ontology, which in computer science is considered as an attempt of comprehensive and detailed formalization of some knowledge domain with the help of conceptual scheme.

\subsection{Ontology Structure}

Structure of the proposed Ontology step-by-step. Class Complex characteristics are used for the description of complex organ characteristics. It is related with class Organ, and more precisely, with some its subclasses, depending on the organ which is described by respective characteristic.

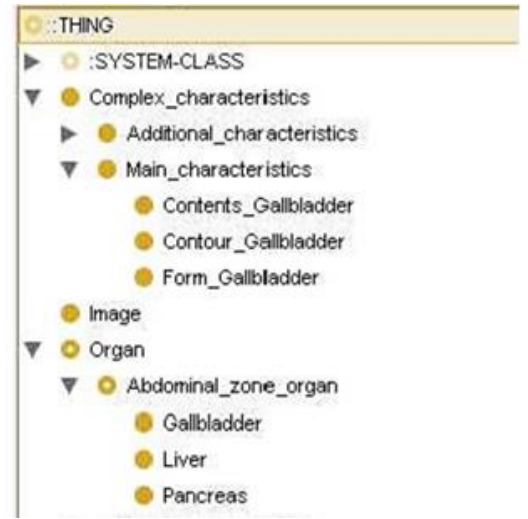

Figure 7. Class Hierarchy [adapted from [27]]

\subsection{Basic Class Organ}

Class Organ is described by such slots as: organ name, region, pathology, image and patient. Slot organ name serves for the organ name. Slot region indicates the area to which the organ belongs. Slot pathology is related with the class Pathology. Every instance of the concrete organ is related with a certain pathology. Slot image is assigned for the image reflecting pathology, indicated in the slot pathology. Slot patient is related with the class Patient and it is assigned for the list of patients, which have the pathology specified in the slot pathology. Class Organ at present has three subclasses: Abdominal zone organ, Cranial zone organ, Toracic zone organ-depending on the area to 
which the organ can belong. All these subclasses have the same slots, as class Organ, differingonly by the allowed values of some slots. Class Abdominal zone organ at present has 3 subclasses: Gallbladder, Pancreas, Liver.

\subsection{Description of subclass Gallbladder}

In subclass Gallbladder the relation in slot pathology changes. Unlike class Organ, the type of slot pathology is declared as"Class with super class Gallbladder pathology". New slots are added in subclass Gallbladder using the knowledge tree as basis. This Knowledge tree is the representation of knowledge about gallbladder for expertssonographists in Expert-shell of SonaRes system.

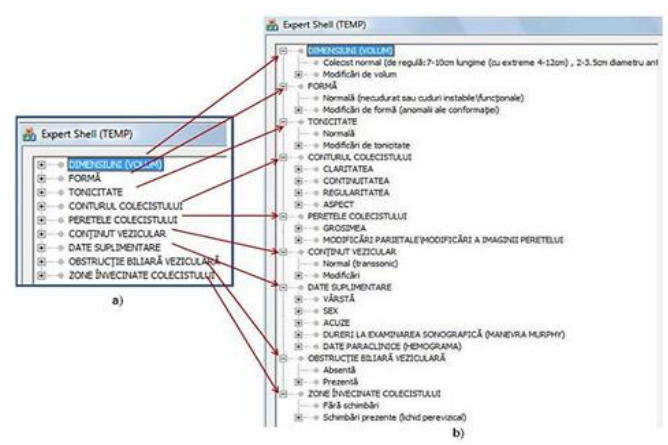

Figure 8. Knowledge tree for Gallbladder in Expertshell:

a) collapsed nodes for characteristics: b) partly expanded nodes for characteristics [27]

In the class Main characteristics create a subclass Contour Gallbladder, and in the class Gallbladder introduce slot contour gallbladder, which is related with class Contour Gallbladder (the slot has the type" instance of Contour Gallbladder"). As shown in Figure 9. In the class Contour Gallbladder the following slots are introduced:

- Slots which correspond to characteristics from knowledge tree: aspect, clarity, continuity, regularity. These slots have allowed values in compliance with the knowledge tree.

- Slot pathology is related with the class Gallbladder pathology. So, its type is "Class with super class Gallbladder pathology". Here those pathologies will be introduced, for the description of which the respective combination of slots values in Contour Gallbladder takes part.

- Slot contour description is the string, in which perse is written somewhat like the title of the respective contour.

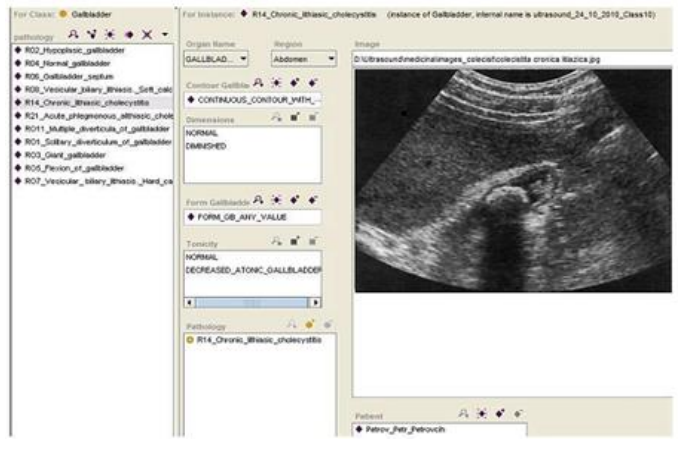

Figure 9. Knowledge on Gallbladder pathology "Chronic lithiasic cholecystitis" [27]

The goal of this research, the process of Ontology elaboration for gallbladder ultrasound images on the base of knowledge enclosed in decision support system SonaRes is described.

\section{Pancreas Based Systems}

In [31], the authors presented Ultrasound investigation (USG) or sonography is currently the leader among the diagnostic methods. Ultrasound investigation has a number of undeniable advantages in comparison with other methods:

- It is the most widespread and effective method;

- The equipment cost for ultrasound investigation is much lower than for other investigation methods (magnetic resonance imaging, computerized tomography, digital radiography, and so on);

- It is a noninvasive method of investigation, has a high diagnostic accuracy and stands out among the other methods due to its informativeness, quickness, reliability and painlessness of investigation.

The knowledge base of the SonaRes system was created during long-term cooperation of physiciansexperts with computer science researchers and represents formal representation of experience and knowledge of physicians in the domain [32]. In fact, this knowledge base is an Ontology [33], but for their goals, they had to present it in one of the standard formats. They have constructed Ontology using the editor of Ontologies Protégé [34]. In this case, they have not moved the whole structure and data, but in the course of development the modifications and corrections concerning the structure of the Ontology were made.

Solution of the problem of building of the Ontology of ultrasound images (UI) is based on the fact that all UI of patients' organs have verbal descriptions. These descriptions are made with the help of medical terms - concepts and characteristics, among which there are the relations and dependencies. Thus, the problem of building of the 
Ontology of UI turns to the task of building an Ontology of UI descriptions (UID), which are associated with the appropriate UI. As shown in Figure 10.

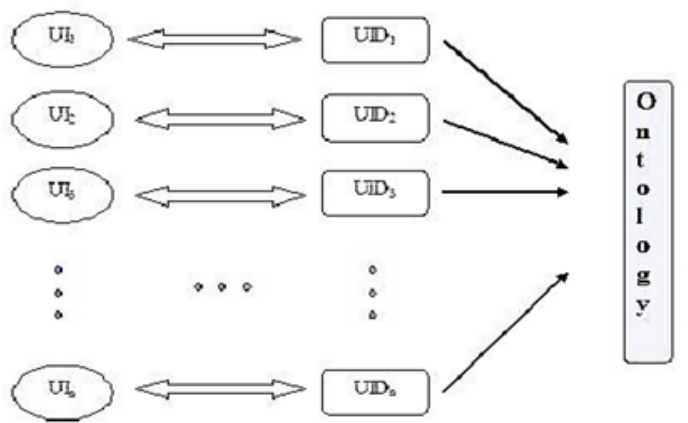

Figure 10. Correspondence between ultrasound images and their descriptions in building the UI Ontology [31]

The goal of this research is to present a method of context-driven annotation in images of the DICOM standard [35] and its application for ultrasound images. Here is described them attempt to create a mapping between the Classification of Diseases ICD-10 [36], and the Ontology of Ultrasound Images (OUI) of hepato-pancreato-biliary zone organs [37].

In [38], the authors mainly focus on the Ontological definition of disease with related properties. The proposed a definition of a disease involving capturing a disease as a causal chain of clinical disorders and a computational model called River Flow Model of Disease [39]. Based on the model, they developed a disease Ontology, which includes definitions of about 6,000 diseases with causal relations between 17,000 clinical disorders (abnormal state). This demonstration shows a system to browse causal chains defined in the disease Ontology.

\subsection{River Flow Model of Disease}

It begins to exist, a typical disease, as a dependent continuant, enacts extending, branching, and fading processes before it disappears. Thanks to these processes, a disease can be identified as a continuant that is anenactor of those processes. Such an entity (a disease) can change according to its phase while keeping its identity. On the basis of this observation, they defined a disease as:

\section{Definition 1: Disease}

A disease is a dependent continuant constituted of one or more causal chains of clinical disorders appearing in a human body and initiated by at least one disorder.

\section{Definition 2: Core Causal Chain of a Disease}

A sub-chain of the causal chain of a disease whose instances are included in all the individual chains of all instances of a particular disease type. It corresponds to the essential property of a disease type.

\section{Definition 3: is-a Relation between Diseases}

Disease $A$ is a super type of disease B if the core causal chain of disease $A$ is included in that of disease B. The inclusion of nodes (clinical disorders) is judged by taking an is-a relation between the nodes into account, as well as the sameness of the nodes. Figure. 11 shows the main types of diabetes constituted by corresponding types of causal chains. Assume, for example, that (non-latent) diabetes and type-I diabetes is respectively defined as <deficiency of insulin-> elevated level of glucose in the blood $>$ and <destruction of pancreatic beta cells->lack of insulin I in the blood-> deficiency of insulin-> elevated level of glucose in the blood $>$. Then, they get <type-I diabetes is-a (non-latent) diabetes> according to Definition 3.

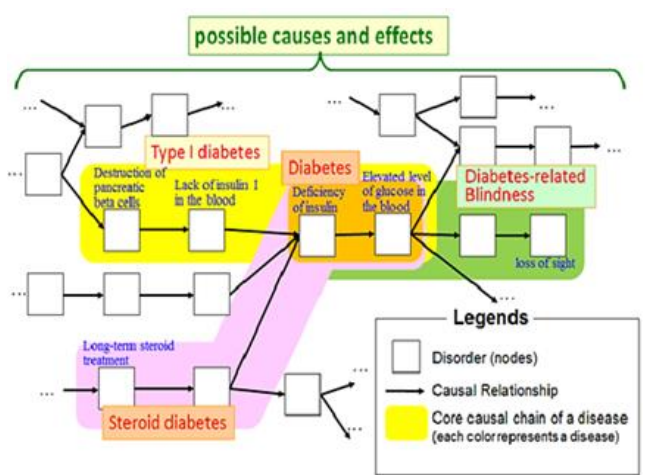

Figure 11. Types of Diabetes constituted of Casual Chain [38]

The goal of this research is to develop a disease Ontology based on River Flow Model and a browsing tool for causal chains defined in it. Because the Ontology is based on Ontological consideration of causal chains, it could capture characteristics of diseases appropriately. The definition of disease as causal could be also very friendly to clinicians since it is similar to their understanding of disease in practice. Moreover, it could include richer information about causal relationships in disease than other disease Ontologies or medical terminologies such as SNOMED-CT.

In [40], the authors presented study how to complement the translation process of the COMMODITY $_{12}$ mediator with Ontologies and semantic reasoning support. The objective is to identify the relevant architecture, tools and techniques to support the development of Ontologies so that it can be integrated into their PHS. They are particularly interested in reasoning about information accessed from the patient database or sensor devices in general, and information regarding data exchanges 
relevant to the profile of a diabetic patient in particular.

\subsection{COMMODITY12 extended with Semantic Reasoning}

COMMODITY12 (Continuous Multi- parametric and Multi- layered analysis Of Diabetes Type 1\&2) aims to design, build, and validate an intelligent system for the analysis of multi-parametric medical data. It twills up take the existing cutting- edge technologies and extend these technologies by combining state-of-the-art networks, software interoperation, and artificial intelligence techniques in order to realize the concept of translational medicine by means of a PHS. As shown in Figure 12.

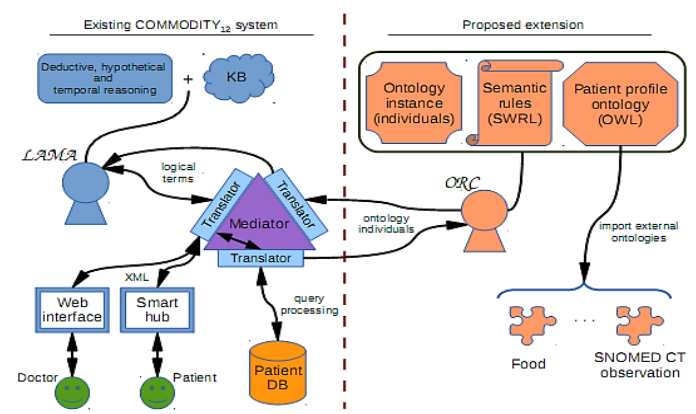

Figure 12. Architecture showing how to extend the COMMODITY $_{12}$ PHS with Ontologies and ORC agent to support semantic reasoning for diabetes patient profiles [40]

- An OWL Ontology describing diabetes patient profiles and importing other relevant Ontologies such as food and medical observations;

- Patient data asserted to the Ontology as OWL individuals, e.g., age, living place, blood pressure measurements;

- Asset of SWRL rules that are used to infer new properties about existing individuals;

- A processing component that retrieves patient information and infers Ontology properties. Currently, ORC agents are purely reactive in that they only respond to requests coming from LAMA agents. When such a request comes regarding the retrieval of specific patient profile information, ORC first loads the Ontology and retrieves relevant data from the patient DB. This Data is then translated to OWL syntax and asserted to the Ontology as individuals. The next step is to execute the SWRL rules to infer new properties about the individuals. These are then translated to logical terms and passed to the LAMA agent that needs those terms for further reasoning.

\subsection{An Ontology for Diabetes Patient Profiles}

The approach is patient centric and aims to include only the necessary patient information so that different types of diabetes can be managed via a
PHS. In other words, the schema defining the Ontology is that of defining a patient profile.

The goal of this research, the authors presented an Ontology reasoning component (ORC) that builds upon existing Ontology modeling tools and techniques to support the integration and interpretation of multimodal medical information. He had illustrated how to embed ORC as a reasoning capability in reactive infrastructure agents supporting intelligent agents operating in COMMODITY 12 , a personal health environment for diabetic patients and the medical professionals that treat them. In figure 12 presents architecture showing how to extend the COMMODITY $_{12}$ PHS [41], with Ontologies and ORC agents to support semantic reasoning for diabetes patient profiles.

In [42], the authors developed the recommendation system of anti-diabetic medication is developed for doctors to use. The experimental data are the diabetic patient's conditions and the endocrinologist's expertise must be trustable. The recommendation system for anti-diabetic drugs are used to recommend drugs more suitable, it avoids the doctor conditional. The restrictions on anti-diabetic drugs are expectant decline in HbA1c (Glycated hemoglobin) levels, safety, side effects, tolerance, convenience, long-term compliance and other effects beyond low-ering blood sugar.

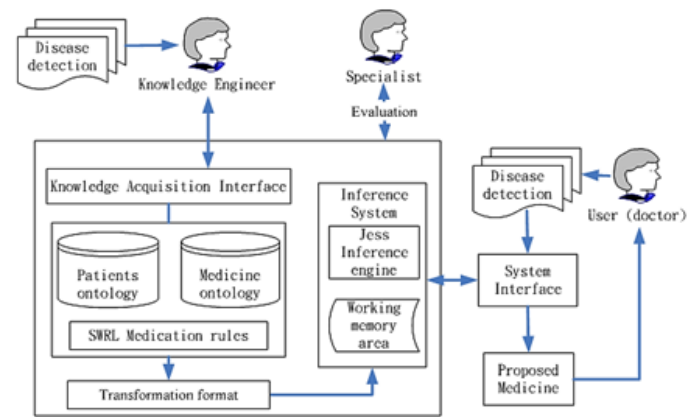

Figure 13. The structure of recommendation system for anti-diabetes drugs [42]

-The knowledge base of anti-diabetic medicine Ontology and patients test Ontology were used to support the normative framework for Ontology. Protégé was used to build the anti-diabetic medicine Ontology to store the regulations of anti-diabetic medicine. The main constituent elements of the Ontology are classes, attributes, and relationships.

-SWRL is an Ontology-based rule language. The rules of medicine regulations were retrieved from the AACEMG. The rules indicate the following: which or alhypoglycemic agent may be used when the HbA1c level is between $6.5 \%$ and $7.5 \%$. What the side effects are, what the contraindication is, and what needs to be monitored will be. 
-Through the inference engine (Pellet), the knowledge class and concept explanation in antidiabetic medicine Ontology were translated into the format that the recommendation system can accept, and then the medical instance may go into operation. The reason is that the instance in Ontology cannot be used when making real inferences about rules. In addition, the inference engine recognizes the conflicting and contradictory knowledge in the Ontology.

-Since SWRL cannot do direct operation, the format needs to be changed through XSLT. However, Protégé3.4.4 established SWRL directly and created rules, which were converted through SWRL 2JESS to JESS in an acceptable format for this study.

-The information, knowledge, experience, and informational rules generated during the process of reasoning were stored in the working memory area temporarily.

-JESS is used for reasoning to identify which agents meet the test results. The instance of contraindication, side effects, and what needs to be monitored may be displayed.

-The user's system aims to provide the doctor with an application interface. A graphical communication interface lets users operate the system easily.

The goal of the research, the authors developed a Diabetes Medication Recommendation system, based on domain Ontology that employ the knowledge base provided by a hospital specialist in Taichung's Department of Health and the database of the American Association of Clinical Endocrinologists Medical Guidelines for Clinical Practice for the Management of Diabetes Mellitus (AACEMG). By thorough analysis, the system first builds ontology knowledge about the drugs' natural attributes, type of dispensing and side effects, and ontology knowledge about patients' symptoms. It then utilizes Semantic Web Rule Language (SWRL) and Java Expert System Shell (JESS) to induce potential prescriptions for the patients. This system is able to analyze the symptoms of diabetes as well as to select the most appropriate drug from related drugs.

In [43], the authors purposed is to offer through three simple stages a solution based on Ontologies to provide personalized monitoring services for patients with any of a wide range of chronic conditions in a tele-monitoring scenario. Presenting the work through the three stages, actions involved in each stage are clearly described enhancing its understanding, reusability and transferability of both the Ontology and the methodology for different domains or applications.

In [44], the authors developed Ontology for the care of chronically ill patients and implement two personalization processes and a decision support tool. The first personalization process adapts the contents of the Ontology to the particularities observed in the health-care record of a given concrete patient, automatically providing a personalized Ontology containing only the clinical information that is relevant for health-care professionals to manage that patient. The second personalization process uses the personalized Ontology of a patient to automatically transform intervention plans describing health-care general treatments into individual intervention plans. For comorbid patients, this process concludes with the semi-automatic integration of several individual plans into a single personalized plan. Finally, the Ontology is also used as the knowledge base of a decision support tool that helps health-care professionals to detect anomalous circumstances such as wrong diagnoses, unobserved comorbidities, missing information, unobserved related diseases, or preventive actions.

\section{Comparative among Ontology-based System for Hepatobiliary System Diseases}

There are many research works described different types of diseases and Ontology-based approach on Hepatobiliary system. These works have been achieved to build specific domain Ontologies and systems for different diseases in Hepatobiliary system as shown in table 2 .

Table 2. Comparison among Ontology-based systems for Hepatobiliary System Diseases

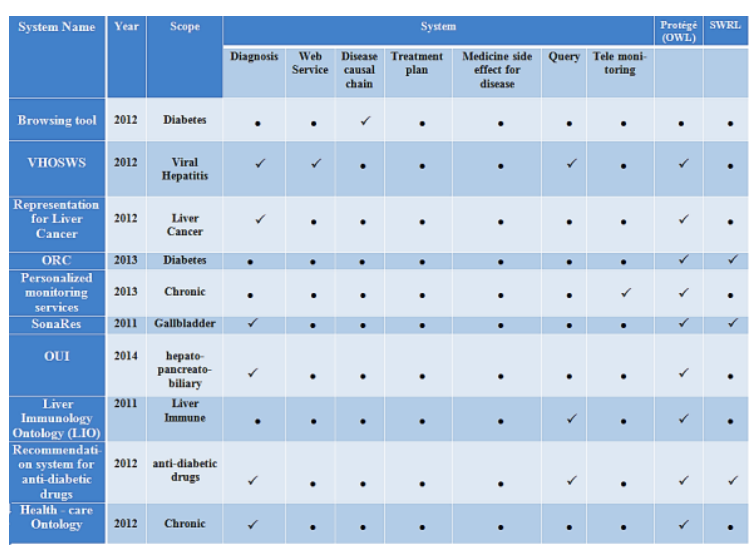

Browsing tool is system for disease casual chain in diabetes. VHOSWS is a web service system for viral hepatitis diagnosis and query by using Ontology, which is built by protégé editor (OWL file). Representation for Liver Cancer is system for liver cancer diagnosis by using Ontology, which is built by protégé editor (OWL file). Ontology reasoning component (ORC) is an Ontology in diabetes field which is built by protégé editor (OWL file) using Semantic Web Rule Language (SWRL). 
A personalized monitoring service is system for chronic tele- monitoring which is built by protégé editor (OWL file). While, SonaRes is a system for Gallbladder diagnosis, which is built by protégé editor (OWL file). Ontology of Ultrasound Images (OUI) is a system for hepato-pancreato-biliary zone organs diagnosis, which is built by protégé editor (OWL file). Liver Immunology Ontology (LIO) is a system for LIO query which is built by protégé editor (OWL file). Recommendation system based for antidiabetic drugs diagnosis and query which is built by protégé editor (OWL file) using SWRL. On the other hand, health-care Ontology is system for chronic diagnosis, which is built by protégé editor (OWL file).

\section{Conclusion}

This paper discussed the current studies on Ontology-based approach and Ontology techniques for Hepatobiliary system diseases. It presented a conceptual view of Ontology based Hepatobiliary system and its infected diseases. Furthermore, it presented a proposed a new classification schema for the research efforts investigated so far based on the Hepatobiliary system organs: Liver, Gallbladder, Bile duct and Pancreas. Besides, the paper shows that the researchers worked in vary systems and Ontology-based approach on Hepatobiliary system.

\section{References}

[1] Cicortas, A., Iordan, V., Fortis, A,"Considerations on Construction Ontologies", Journal Annals Computer Science Series 1, 79-88, 2009.

[2] W3C XML Schema Definition Language, http://www.w3.org/TR/xmlschema11-1/, (last visited 12 Oct, 2014.

[3] Resource Description Framework (RDF) Schema Specification. http://www.w3.org/TR/PR-rdf-schema/, (last visited 29 Oct, 2014).

[4] DAML+OIL Web Ontology Language. http://www.w3.org/Submission/2001/12/, (last visited 29 Oct, 2014).

[5] Web Ontology Language (OWL) Overview. http://www.w3.org/TR/owl-features/, (last visited 29 Oct, 2004).

[6] Zhanjun Li, Maria C. Yang and Karthik Ramani, "A methodology for engineering ontology Acquisition and validation", Artificial Intelligence for Engineering Design, Analysis and Manufacturing, PP: 37-51, USA, 2009.

[7] Moise Gabriela, Netedu Loredana, "Ontologies for Interoperability in the eLearning Systems", Bulletin of Petroleum-Gas University of Ploiesti Mathematics, Informatics, Physics Series [BMIF], ISSN 1224-4899, EISSN 2067-242X, Volume LXI No. 2, PP: 75-88, 2009.
[8] Youn Seongwook, Arora Anchit, Chandrasekhar Preetham, Jayanty Paavany, Mestry Ashish and Sethi Shikha, "Survey about Ontology Development Tools for Ontology-based Knowledge Management", University of Southern California, 2009.

[9] H. Pundt and Y. Bishr, "Domain Ontologies for Data Sharing-An Example from Environmental Monitoring Using Field GIS", Computer \& Geosciences, 28, PP: 98102, 1999.

[10] Protégé. http://protege.stanford.edu/, (last visited 29 Oct, 2012).

[11] Manolis Maragoudakis and Ilias Maglogiannis, "A medical ontology for intelligent web-based skin lesions image retrieval", Journal Health Informatics Journal, PP: 140-157, 2011.

[12] Sánchez, D., Moreno, A., "Learning Medical Ontologies from the Web", LNCS Knowledge management for Health care Procedures, Vol. 4924, PP: 32-45, 2008.

[13] Smith B, Ceusters W, Klagges B, Köhler J, Kumar A, Lomax J, Mungall C, Neuhaus F, Rector AL \& Rosse C, "Relations in biomedical ontologies", Genome Biology, Vol. 6, 2005.

[14] Samantha Bail, Matthew Horridge, Bijan Parsia, Ulrike Sattler, "The Justificatory Structure of the NCBO BioPortal Ontologies", Proceeding of The Semantic Web ISWC, Vol. 7031, PP: 67-82, 2011.

[15] C. Paul Morrey, James Geller, Michael Halper \& Yehoshua Perl, "The neighborhood auditing tool: A hybrid interface for auditing the UMLS", Journal of biomedical informatics, Vol. 42, PP: 468-489, 2009.

[16] Mythili Thirugnanam, Mangayarkarasi Ramaiah, V Pattabiraman, R Sivakumar, "Ontology based Disease Information System", International Conference on Modelling Optimization and Computing, PP: 3235- 3241, 2012.

[17] Official Partner of the Liver Strong Foundation, http://www.livestrong.com/article/119869-list-body-systems, (last visited 10 December, 2014).

[18] Medicine Net, http://www.medicinenet.com/ liver_disease/article.htm/, (last visited 10 December, 2014).

[19] http://hepatitis.about.com/od/jkl/g/liver.htm/. (last visited 10 December, 2014).

[20] Moawad,I, Al Marzoqi,G, Salem, A," Web Service Based Approach for Viral Hepatitis Ontology Sharing and Diagnosing", In Proceeding of AMLTA, PP: 257-266, Springer-Verlag Berlin Heidelberg 2012.

[21] Marco Alfonse, Mostafa M. Aref, Abdel-Badeeh M. Salem. "Ontology-Based Knowledge Representation for Liver Cancer". Proceedings of the International eHealth, Telemedicine and Health ICT Forum for Educational, Networking and Business. Luxembourg, G. D. of 
Luxembourg, ISSN 1818 -9334, PP: 821-825, April 18-20, 2012.

[22] M. Dean, G. Schreiber. "OWL Web Ontology language Reference", W3C Working Draft, http://www.w3.org/TR/owl-ref/. 2003.

[23] Knublauch, H., et al., The Protégé OWL plugin: An open development environment for semantic web applications. The Semantic Web-ISWC 2004, PP: 229243, 2004.

[24] MedicineNet.com, http://www.medicinenet.com/ script/main/hp.asp. (last visited 13 January, 2015).

[25] Cancer.Net, http://www.cancer.net/portal/site/patient. (last visited 17 January, 2015)

[26] Anna Maria Masci, Jeffrey Roach, Bernard de Bono, Pierre Grenon, Lindsay Cowell, "Bridging multiple Ontologies Representation of the liver Immune Response", International Conference on Biomedical Ontologies (ICBO), Buffalo,NY, USA, Working with multiple Biomedical Ontologies Workshop, 2011.

[27] Natalie Bruc, GalinaMagariu, TatianaVerlan," Gallbladder Description in Ultrasound Images Ontology", Proceeding of Modelling and Development of Intelligent Systems, PP: 18-27, Sibiu-Romania, 2011.

[28] L.Burtseva, S.Cojocaru, C.Gaindric, E.Jantuan, O.Popcova, I.Secrieru, D.Sologub, SONARES-A Decision Support System in Ultrasound Investigations. Computer Science Journal of Moldova, Vol.15, PP: 153-177, 2007.

[29] O. Popcova, S. Puiu, Iu. Secrieru, "Knowledge Structurization and Formalization for Gallbladder Ultrasound Investigation Domain". Proceedings of the IIInternational Conference on Advanced Information and Telemedicine Technologies for Health, Minsk, Belarus, PP: 98-102, 2008.

[30] N. Bruc, "Knowledge Representation with theHelp of Ontologies in SonaRes System", Collection of Abstracts of BIT+ "Information Technologies-2008", 2008, Chisinau, Vol.8, PP..50-53, in Russian.

[31] Natalia BRUC, "An approach to mapping between the classification of diseases ICD-10 and the Ontology of Ultrasound Images of hepato-pancreato-biliary zone organs", 8th International Conference on Microelectronics and Computer Science, Chisinau, Republic of Moldova, PP: 314-317, 2014.

[32] Burtseva L., Cojocaru S., Gaindric C., Jantuan E., Popcova O., Secrieru I., Sologub D. "SONARES - A Decision Support System in Ultrasound Investigations", CSJM, - N 2 (44), - Vol. 15, PP: 153-177, 2007.

[33] Iulian Secrieru, "SonaRes methodology enhancement using knowledge discovery technique", Proceedings of the Third Conference of Mathematical Society of Moldova IMCS-50, Chisinau, ISBN 978-9975-68-244-2, PP. 557 $562,2014$.
[34] Ontology editor Protégé, Available: http://protege. stanford.edu/. (last visited 18 December 2014).

[35] DICOM, Available: http://dicom.nema.org/, (last visited 12 January, 2015).

[36] International Statistical Classification of Diseases and Related Health Problems 10th Revision (ICD-10), Available: http://apps.who.int/classifications/icd10/

browse/2010/en\#/K80-K87, last visited 4 December, 2014.

[37] N. Bruc, G. Magariu, T. Verlan, "Elaborating of Ultrasound Images Ontology in Ultrasound Diagnostics", in the International Conference on e-Health and Bioengineering, EHB, Iasi, In CD, Gr.T.Popa University of Medicine and Pharmacy Publishing House, Iaşi, România, Editors: Hariton Costin, Alexandru Morega, Liliana Vereştiuc. ISBN: 978-606-544-078-4, 2011.

[38] Kouji Kozaki, Hiroko Kou, Yuki Yamagata, Takeshi Imai, Kazuhiko Ohe, Riichiro Mizoguchi," Browsing Casual Chain in a Disease Ontology", International Semantic Web Conference (Posters \& Demos)'12, 2012.

[39] Kozaki, K., et al. "Identity Tracking of a Disease as a Causal Chain”, Proceedings of ICBO2012. 2012.

[40] Kafali, Ozgur; Sindlar, Michal; Weide, Tom van der; Stathis, Kostas, "ORC: an Ontology Reasoning Component for Diabetes", 2nd International Workshop on Artificial Intelligence and Netmedicine (NetMed'13). 2013.

[41] Kafal1, Ö., Bromuri, S., Sindlar, M., van der Weide, T., Pelaez, E.A., Schaechtle, U., Stathis, K, "COMMODITY12: A smarte-health environment for diabetes management", Journal of Ambient Intelligence and Smart Environments, IOS Press (Toappear), 2013.

[42] Rung-Ching Chen, Yun-Hou Huang, Cho-Tsan Bau, Shyi-Ming Chen," A recommendation system based on domain ontology and SWRL for anti-diabetic drugs selection", Expert Systems with Applications, Volume 39, Issue 4, PP: 3995-4006, 2012.

[43] Lasierra N., Alesanco A, Guillén S, García J, “A three stage ontology-driven solution to provide personalized care to chronic Patients at home", Journal of Biomedical Informatics, PP: 516-529, 2013.

[44] David Riaño, Francis, Joan Albert López-Vallverdú, Fabio Campana, Sara Ercolani, Patrizia Mecocci, Roberta Annicchiarico, Carlo Caltagirone," An ontology-based personalization of health-care knowledge to support clinical decisions for chronically ill patients", Journal of Biomedical Informatics Volume 45, Issue 3, PP: 429-446, 2012. 